Seguridad Operacional

y Logística Aeronáutica

Segurança Operacional

e Logística Aeronáutica

Operational Safety

and Aviation Logistics

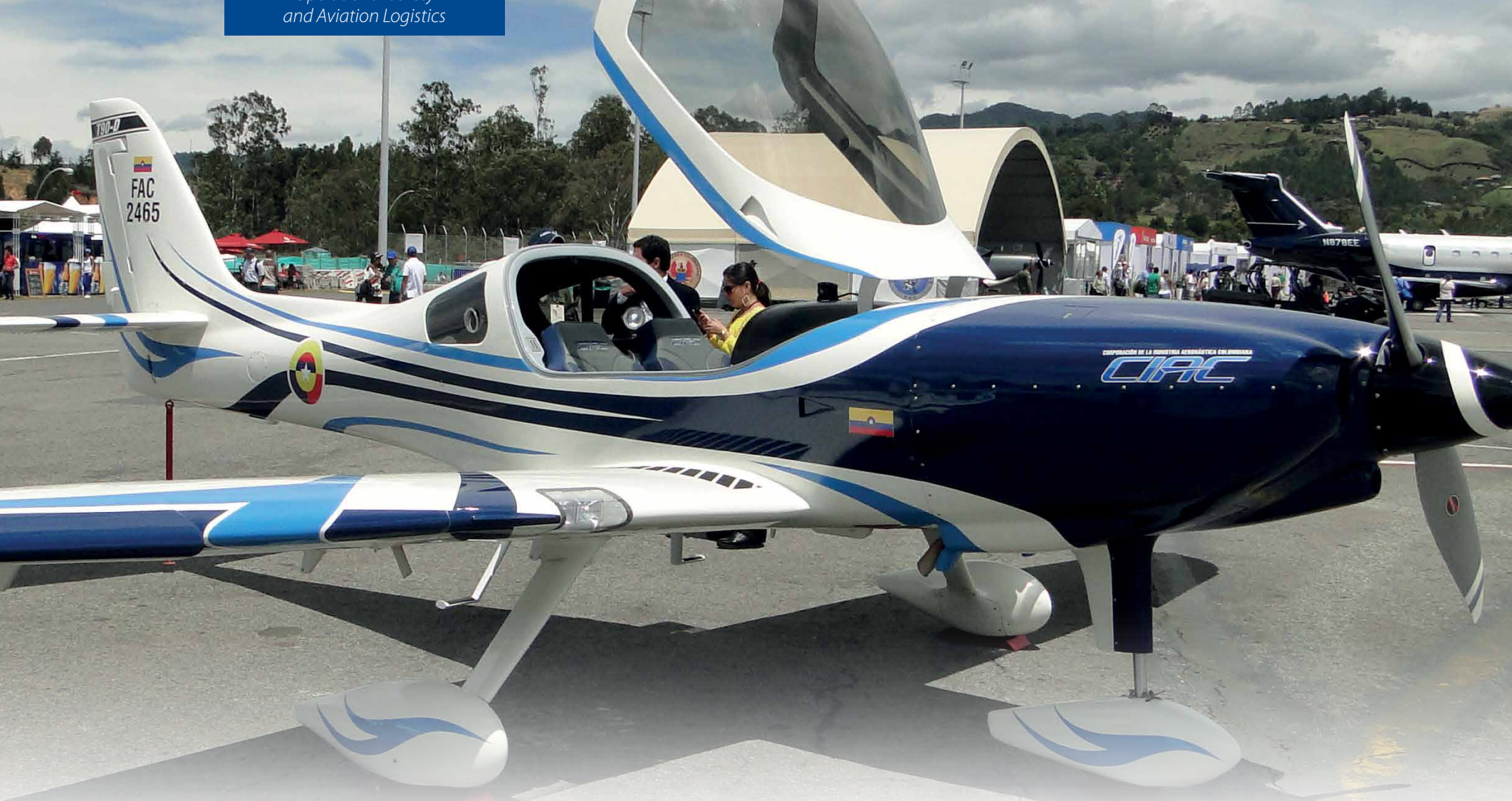

\title{
Propuesta para disminuir la temperatura en la Cabina del avión T-90 Calima
}

Proposta PARA REDUZIR A TEMPERATURA NA CABINE DO AVIÃO T90-CALIMA²

Proposal to Reduce Temperature in the Aircraft Cabin of T90-Calima ${ }^{3}$

Néstor Fabián Cedeño Niño, Elías Fernando Acosta Palacios, Ramiro Alejandro Plazas Rosas Escuela Militar de Aviación Marco Fidel Suárez

\section{CIENCIA Y PODER AÉREO}

ISSN 1909-7050 / E- ISSN 2389-2468 / Volumen 10 / Enero-Diciembre de 2015/ Colombia/ Pp. 1-260 Recibido: 23/09/2015

Aprobado evaluador interno: 12/10/2015

Aprobado evaluador externo: 20/11/2015

Doi: http://dx.doi.org/10.18667/cienciaypoderaereo.190 
CIENCIA Y PODER AÉREO | Revista Científica de la Escuela de Postgrados de la Fuerza Aérea Colombiana | Vol. 10 | Enero - Diciembre de 2015

Para citar este artículo:

Cedeño, N., Acosta, E., y Plazas, R. (2015). Propuesta para disminuir la temperatura en la cabina del avión T-90 Calima. Ciencia y Poder Aéreo, 10 (1), 49-58. Doi: http://dx.doi. org/10.18667/cienciaypoderaereo.190

'Artículo científico original, realizado en el marco del proyecto Estrategias para la promoción e impulso para el desarrollo de procesos investigativos en la Escuela Militar de Aviación "Marco Fidel Suárez", del Grupo de Investigación en Estudios Aeroespaciales - GIEA, EMAVI CP-01-PIMEC-2015.

${ }^{2}$ Artigo científico original, realizado no âmbito do projecto estratégias para a promoção e impulso para o desenvolvimento de processos de pesquisa nas Escola Militar de Aviação "Marco Fidel Suárez" Grupo de Pesquisa Estudos Aeroespaciais - GIEA, EMAVI CP-01-PIMEC -2015.

${ }^{3}$ Original scientific article from the research Project: Strategies for the promotion and impulse for the development of research processes in the Aviation Military School "Marco Fidel Suarez" Research Group of Aerospace Studies. -GIEA-EMAVI CP-01-PIMEC-2015.

${ }^{4}$ Subteniente, Ingeniero mecánico, Escuela Militar de Aviación "Marco Fidel Suárez". Cali, Colombia. Correo electrónico: nefacenio@gmail.com

${ }^{5}$ Subteniente, Ingeniero mecánico, Escuela Militar de Aviación "Marco Fidel Suárez". Cali, Colombia. Correo electrónico: fercho9091@gmail.com

${ }^{6}$ Ingeniero electrónico; Magíster en ingeniería énfasis automática. Coordinador -Sección investigaciones Escuela Militar de Aviación "Marco Fidel Suárez". Cali, Colombia. Correo electrónico: ramiroplazas@hotmail. com
Resumen: la temperatura es un factor importante en el momento de realizar una labor; sí la sensación térmica no es agradable, un trabajo sencillo puede transformase en un trabajo complicado, donde se puede exponer la integridad de la persona. En las aeronaves de entrenamiento los pilotos no son ajenos a los inconvenientes de la exposición a una temperatura elevada generada por radiación solar, equipos, entre otros. Al incluir un sistema de refrigeración a la cabina de entrenamiento de la aeronave ésta se ve afectada en características como equilibrio, mayor consumo de combustible, disminución de la eficiencia debido al aumento de peso. En este artículo, se proponen tres formas de disminuir la temperatura en la cabina del avión T-90, en las diferentes fases de vuelo, que consisten en implementar tomas y salidas de aire, utilizar una película térmica para la cabina y desarrollar un dispositivo que permita la circulación de aire en la fase previa al despegue.

Palabras clave: aeronave; carlinga; temperatura.

Resumo: a temperatura é um fator importante no momento de realizar uma atividade, se a sensação térmica não é agradável, um trabalho simples pode se transformar num trabalho complicado, o que poderia expor a integridade da pessoa. Nas aeronaves de treinamento, os pilotos não são estranhos aos inconvenientes de exposição a uma temperatura elevada gerada pela radiação solar, equipamentos, entre outros. Ao incluir um sistema de refrigeração à cabine de treinamento da aeronave, esta se ve afetada nas características de equilíbrio, aumento do consumo de combustível e diminuiçao da eficiência, devido ao ganho de peso. Neste artigo, propõem-se três maneiras de reduzir a temperatura na cabine da aeronave T-90 nas diferentes fases do voo, que consistem em implementar as entradas e saídas de ar, usar um filme térmico para a cabine e em desenvolver um dispositivo que permita a circulação de ar no pré-decolagem.

Palavras-chave: aeronave; cabine do piloto; temperatura.

Abstract: Temperature is important when performing an activity, if wind chill is not pleasant, a simple job can turn into a complicated job, exposing the integrity of the person. In training aircrafts, pilots are aware of the inconveniences of exposure to high temperature generated by solar radiation and equipment among others. By including a cooling system on the training cabin, the aircraft is affected by characteristics such as balance, increased fuel consumption and decreased efficiency due to increased weight. In this article, we present three ways to reduce the temperature in the cabin of the aircraft T-90 in the different phases of flight. They consist of implementing air inlets and outlets, using a heating film for the cabin and developing a device that allows air circulation in the pre-takeoff stage.

Key Words: Aircraft; Cockpit; Temperature. 


\section{Introducción}

Los seres humanos tiene una temperatura constante y cuentan con sistemas propios de regulación de temperatura de esta forma se evita que se altere el funcionamiento fisiológico, evitando que se comprometa la salud de la persona. En el trabajo realizado por (Susan C. Kim, MD - Pediatría \& John Pope, 2014) explica que la forma como la temperatura corporal se mantiene, es a través de la sudoración, respiración y variación del ritmo cardiaco. De igual forma, los protocolos de seguridad industrial (Escuela Colombiana de Ingeniería, 2008) recomiendan alejarse de las fuentes de calor para que el trabajador pueda hidratarse y recuperarse. En el trabajo de (Mancera Fernández, 2016), se menciona que la temperatura ideal para realizar un trabajo debe estar entre los $19^{\circ} \mathrm{C}$ y $22^{\circ} \mathrm{C}$.

En diciembre de 2009, se inició la producción de la primera aeronave para entrenamiento primario hecho en Colombia, equipo que para la Fuerza Aérea Colombiana (FAC) recibió la denominación de CALIMA T-90 en honor a los ancestros que ocuparon el Valle del Cauca durante un importante período histórico. La designación «T»por su parte, corresponde a la palabra «training» y el número 90 es referencia conmemorativa a los 90 años de la Fuerza Aérea (CIAC, 2015), con el transcurrir del tiempo las aeronaves fueron sometidas a diversas modificaciones hasta la actual versión conocida como T-90C, que cuenca con mejoras, tales como micro vórtices en los estabilizadores del empenaje, la instalación de Wung Cuff y Stall Strip y una actualización a pantallas Garmin (Garmin Ltd., 2015).

En un estudio realizado por Establecimiento de Sanidad Militar (ESM) (2013), reveló que la tripulación de la aeronave T-90C se encuentra expuesta a una temperatura máxima de $50^{\circ} \mathrm{C}$ y un promedio de $46^{\circ} \mathrm{C}$, sobrepasando el límite de $30^{\circ} \mathrm{C}$ con un porcentaje de humedad relativa de $30 \%$, los cuales son recomendados por el Instituto de Medicina Aeroespacial de los Estados Unidos (Naval Aerospace Medical Institute, 1991).

Bajo las condiciones climáticas de operación en la ciudad de Cali, donde se encuentra parte de la flota de las aeronaves T-90, se registraron temperaturas, humedad relativa y velocidad del aire dentro de la cabina en las diferentes fases del vuelo (Acosta Palacios y Cedeño Niño, 2015). Según los datos registrados, se requiere disminuir la temperatura en la cabina de la aeronave T-90 Calima.

Según una investigación realizada por la NASA (Frick, Davis, Randall, y Mossman, 1945; Alvin, H. S., 1951), para drenar parte del caudal de aire generando la mínima resistencia en la aeronave, desarrollaron tomas de aire de perfil NACA, las cuales son una clase de tomas sumergidas en la pared del avión y por lo general no presentan área frontal externa; las cuales se diseñan para canalizar una parte del flujo alrededor de la aeronave, disminuyendo la temperatura en la cabina.

De esta manera, existe un tipo de ventanillas corredizas, empleadas para permitir el ingreso de aire a la cabina denominadas Ventanillas visa vents, las cuales se adaptan en burbujas de plexiglás (ver Figura 1). En la actualidad, dichas ventanillas son empleadas en varias aeronaves como el monomotor Cosmik EV-97 fabricado por Evektor Aerotechnik (Evektor-Aerotechnik, 2015).

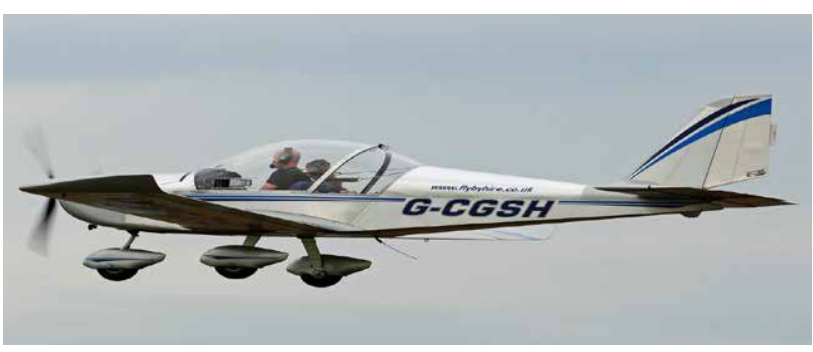

Figura 1. Ventanillas visa vents del Cosmik EV-97.

Fuente: Stephen Blee (2011). Cosmik EV-97 TeamEuroStar UK. Retrieved July 13, 2015, from http://tinyurl.com/nk6svab

Durante la visita geoestratégica que realiza el personal de la Escuela Militar de Aviación "Marco Fidel Suárez" (EMAVI), a la Escuela de la Fuerza Aérea de los Estados Unidos (USAF), se observó el sistema de ventanillas visa vents con las que cuentan los planeadores empleados por la USAF, para dar un primer acercamiento al vuelo a su personal de alumnos. En la Figura 2 se puede observar el sistema de ventanillas visa vents, además de ser corredizas, cuentan con una pestaña que permite el ingreso de aire desplegándose un poco hacia afuera para reducir caudal de aire si así se desea.

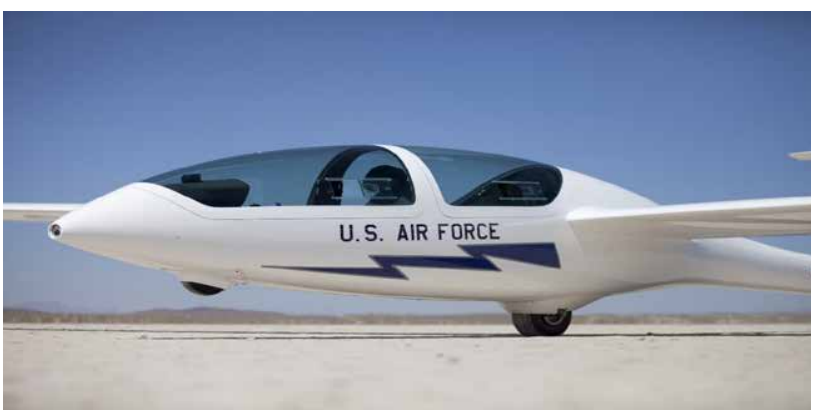

Figura 2. Flugzeugbau DG-1001 Glider.

Fuente: U.S. Air Force Photo by Christian Turner (2011). Flugzeugbau DG-1001 Glider. Retrieved July 15,2015, from http://www. edwards.af.mil/photos/mediagallery.asp?gallerylD=2515\&?id=1 \&page $=4 \&$ count $=48$ 
Debido a la problemática presentada con respecto a la temperatura en la cabina, la Corporación de la Industria Aeronáutica Colombiana (CIAC) como casa fabricante de avión T-90 Calima, inició un estudio para determinan la posición y el perfil más apropiado para canalizar un caudal de aire, desde la capa laminar alrededor del borde de la carlinga basándose en la investigación experimental de Frick et al. (1945).

El desarrollo por parte de la CIAC, consistió en alojar sobre el marco exterior de la carlinga, hecho en material compuesto, un perfil de entrada de aire tipo NACA Scoop (ver Figura 3); se aprovechó el espacio existente en este montaje como conducto. De igual forma, se impermeabilizó buscando prevenir de daños del material compuesto en la estructura del marco y se incluyó un sistema de drenaje para evacuar cualquier deposición de agua en este conducto.

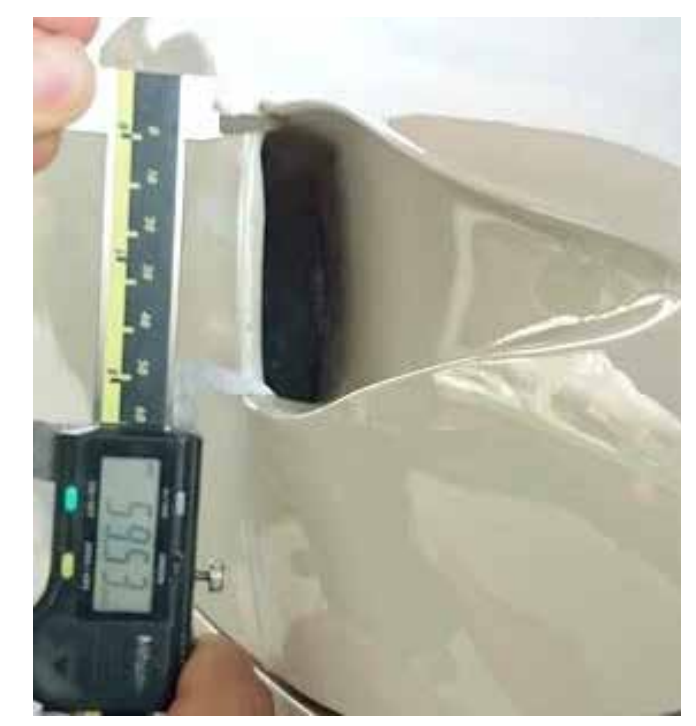

Figura 3. Medidas de la toma NACA Scoop.

Fuente: autores.

Al finalizar el conducto de flujo de aire se encuentra un Eyeball Vent (ver Figura 4) para regular el flujo de aire y 3 grados de libertad en rotación que permiten re-direccionar el flujo. Las entradas añadidas en el marco exterior de la carlinga las cuales en comparación con la entrada de perfil NACA de la OAT son más grandes. Por tanto, canalizan un volumen de aire mucho mayor, logrando de esta manera evacuar gran cantidad de calor del interior de la cabina por convección forzada.

Para permitir la recirculación del aire dentro de la cabina se diseñaron unas salidas de aire como se muestra en la Figura 5; las ventanillas traseras del avión en forma circular cuentan con una tapa para permitir cerrarla. Las ventanillas

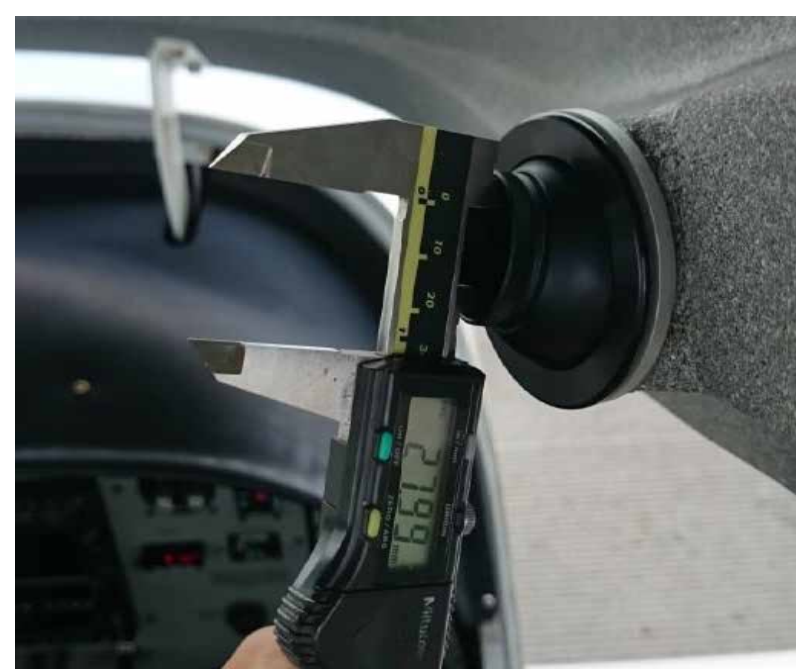

Figura 4. Medidas de Eyeball vent, tomas Canopy. Fuente: autores.

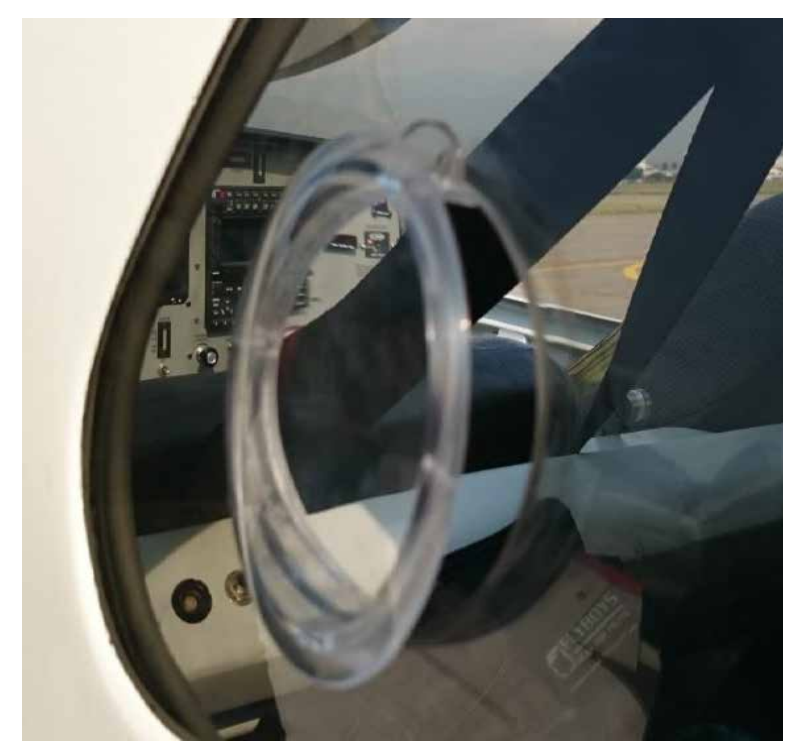

Figura 5. Salida de aire en las ventanillas. Fuente: autores.

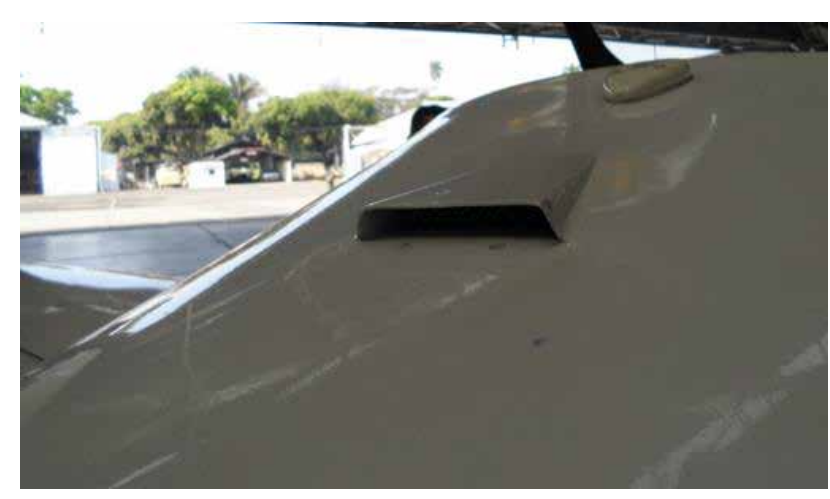

Figura 6. Salida de aire en el fuselaje.

Fuente: autores. 
se hicieron empleando una cierra circular para prevenir la propagación de fisuras en toda la ventanilla. Anteriormente, en la misma aeronave se había instalado una salida de aire en el fuselaje (ver Figura 6).

De acuerdo al trabajo realizado por la CIAC y con el propósito de mejorar el confort térmico en la cabina de avión T-90, se realiza las siguientes propuestas: sistema de climatización por convección forzada, película térmica y soporte de la carlinga para movimientos en tierra.

\section{Método}

\section{Sistema de climatización por convección forzada}

Tomas de aire de perfil NACA. Empleando el proceso para optimizar entradas de perfil NACA (Frick et al., 1945) y con la intención de reducir la resistencia aerodinámica generada por la adaptación de estas tomas en la superficie exterior de la aeronave, se obtuvo las dimensiones adecuadas para el sistema de climatización por convección forzada en la cabina del avión.

Según Frick et al. (1945), para diseñar una toma de aire de perfil NACA es necesario establecer la proporción de ancho y profundidad, teniendo una proporción entre 3 y 5 para reducir las pérdidas de presión; por tal motivo y teniendo por referencia las tomas hechas por la CIAC y la relación de proporción, debe tener $60 \mathrm{~mm}$ de ancho y una profundidad de $15 \mathrm{~mm}$ (sin incluir el espesor del labio o borde posterior de la toma). El radio correspondiente al centro del labio debe ser de 2,82 mm. Los puntos correspondientes a las coordenadas son mostrados en la Tabla 1. La Figura 7 enseña el diseño realizado en Solidworks ${ }^{\circledR}$ (SolidWorks Corp., 2015).

Tabla 1. Medidas del labio para la toma diseñada.

\begin{tabular}{ccc}
\hline \multicolumn{3}{c}{ Forma del labio toma NACA } \\
\hline Posición $(x)$ & $\begin{array}{c}\text { Superficie } \\
\text { superior }(y)\end{array}$ & $\begin{array}{c}\text { Superficie } \\
\text { inferior }(y)\end{array}$ \\
\hline 0 & 2.955 & 2.955 \\
\hline 1,8750 & 1.305 & 4.875 \\
\hline 3,75 & 0.84 & 5.625 \\
\hline 5,625 & 0.54 & 6.18 \\
\hline 7,5 & 0.315 & 6.6 \\
\hline 9,375 & 0.18 & 6.93 \\
\hline 11.125 & 0.09 & 7.215 \\
\hline 13.125 & 0.03 & N/A \\
\hline 15 & 0 & N/A \\
\hline
\end{tabular}

Fuente: elaboración de los autores.

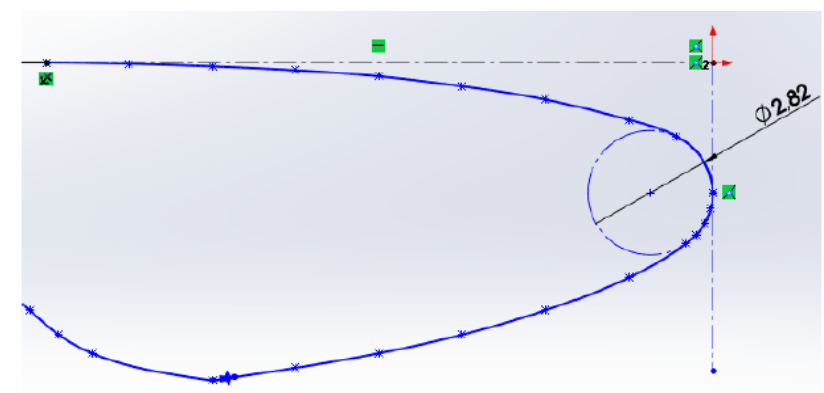

Figura 7. Labio de la toma diseñado.

Fuente: elaboración de los autores. [Solidworks ${ }^{\circledR}$ edición educativa (2012-2013)].

La profundidad total de la toma incluyendo el espesor del labio corresponde a 22,215 mm por tanto para que la rampa mantenga un ángulo de 70 de declinación y prevenir el desprendimiento del flujo laminar la longitud del recorte debe ser de 181,0 mm. La forma del recorte se consiguió recurriendo nuevamente al método de Charles (Frick et al., 1945). En la Figura 8, se observan las diez líneas de construcción que se encuentran acotadas a $18,1 \mathrm{~cm}$ de separación para completar de esta manera la longitud y forma del recorte.

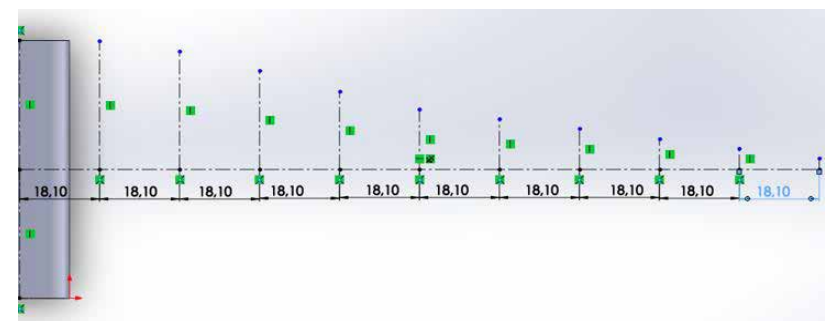

Figura 8. Forma del recorte para la toma del diseño. Fuente: elaboración de los autores. [Solidworks ${ }^{\circledR}$ edición educativa (2012-2013)].

En condiciones atmosféricas ISO y con una velocidad de 65 kt, se realizó una simulación de flujo en Solidworks ${ }^{\circledast}$ (SolidWorks Corp., 2015) para determinar cómo varia el coeficiente de fricción en la superficie y realizando una comparación con los datos obtenidos por la CIAC (Acosta Palacios y Cedeño Niño, 2015). En la Figura 9., se identifica la necesidad de incluir un perfil aerodinámico en el borde posterior de la toma, con el propósito de reducir la fricción en el perfil posterior y prevenir el desprendimiento laminar que generaba turbulencia en la zona posterior a la toma.

Salidas de aire. Para tener una noción del movimiento del aire al interior de la cabina de un avión T-90C estándar se emplearon los planos digitales de la aeronave proporcionados por la CIAC (ver Figura 10), como molde para ge- 


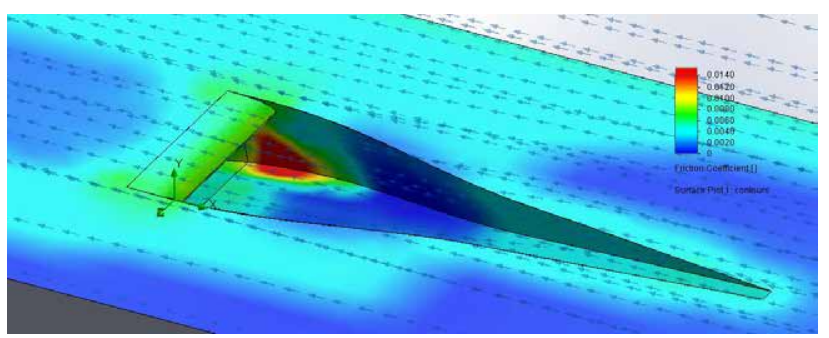

Figura 9. Variación del coeficiente de arrastre en la superficie de la toma diseñada.

Fuente: elaboración de los autores. [Solidworks ${ }^{\circledR}$ edición educativa (2012-2013)].
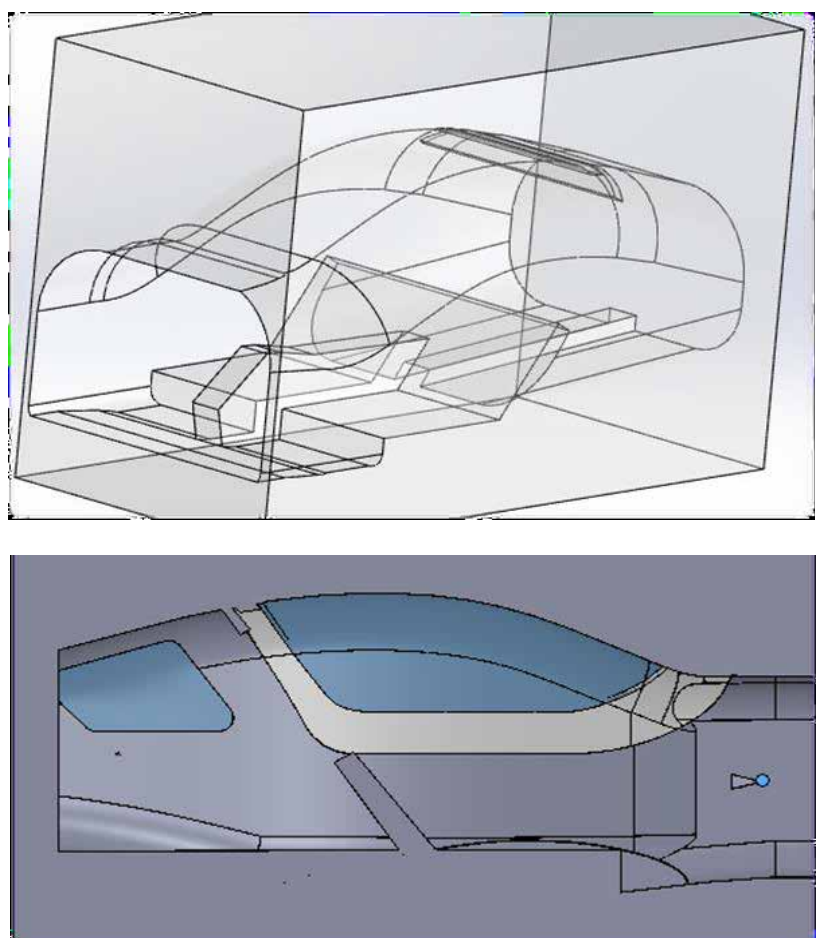

Figura 10. Modelo digital del interior de la cabina. Fuente: CIAC - autores. [Solidworks ${ }^{\circledR}$ edición educativa (20122013)].

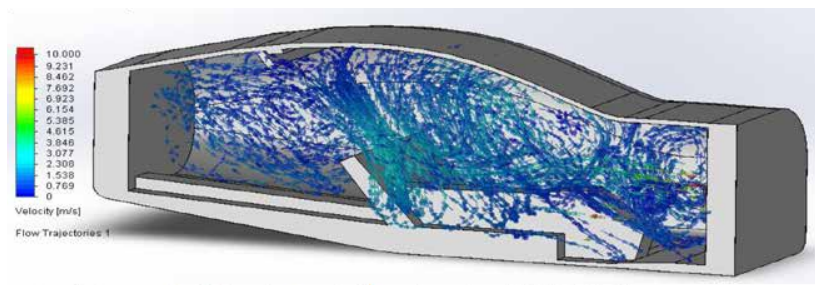

Figura 11. Flujo en la cabina sin salidas de aire. Fuente: elaboración de los autores. [Solidworks ${ }^{\circledast}$ edición educativa (2012-2013)].

nerar el volumen interior de la cabina y graficar las líneas de flujo dentro de la misma durante un despegue a $65 \mathrm{kt}$ de velocidad.
Empleando la herramienta de simulación de fluidos (flow simulation) de Solidworks se genera una vista de sección de la cabina, en la cual se graficaron 15 líneas de flujo como se concluyó en el estudio térmico, (Acosta Palacios y Cedeño Niño, 2015). En la Figura 11 se observa que la velocidad del aire en las entradas OAT se mantiene en un promedio de $10 \mathrm{~m} / \mathrm{s}$ siendo esta la máxima, asimismo, el predominio del tono azul en la Figura indica que el movimiento del aire al interior de la cabina es casi nulo.

Teniendo como referencia las condiciones de la cabina sin salidas de aire se decidió evaluar el comportamiento del flujo al incluir la salida implementada por la CIAC. El movimiento de la masa de aire en el área de los asientos de los tripulantes ascendió de $3 \mathrm{~m} / \mathrm{s}$ a un intervalo entre 3,8 y $6 \mathrm{~m} / \mathrm{s}$ como se puede observar en la Figura 12, esto debido a la liberación de presión en la parte posterior de la cabina tras incluir la salida de aire en la parte superior del fuselaje. No obstante, la renovación completa del cuerpo de aire no se consigue, debido a que parte de fluido permanece en el compartimiento de equipaje del avión, de tal manera que el calor corporal evacuado de los tripulantes permanece en el aire al interior de la cabina, incrementando así la temperatura y el índice de humedad relativa.

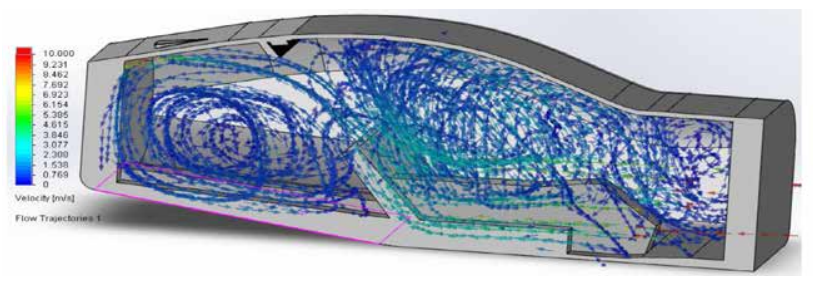

Figura 12. Flujo en la cabina con la salida propuesta por la CIAC. Fuente: autores. [(Solidworks ${ }^{\circledast}$ edición educativa (2012-2013)].

El comportamiento del flujo de aire al incluir unas salidas de aire tipo Louvers mediante la cuales se puede conseguir canalizar el aire desde el interior en varias secciones. Para efectos de simulación se incluyeron dos de estas rejillas con tres perforaciones, cada una con un área de $24 \mathrm{~cm}^{2}$, estas se ubicaron en la parte superior del fuselaje del avión tal como aparece en la Figura 13, siendo allí donde se estanca el flujo de aire y no se compromete estructuralmente la aeronave.

De la simulación de flujo se obtuvo la Figura 14, donde se hace notorio el incremento de velocidad de circulación dentro de la cabina permitiendo de esta manera evacuar rápidamente el aire albergado, y asimismo acelerar la transferencia de calor por convección forzada. 


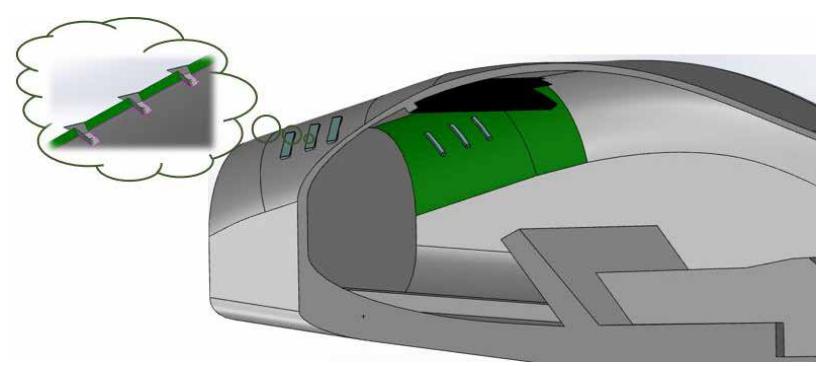

Figura 13. Rejillas Louvers en el fuselaje del T-90C. Fuente: elaboración de los autores. [(Solidworks ${ }^{\circledR}$ edición educativa (2012-2013)].

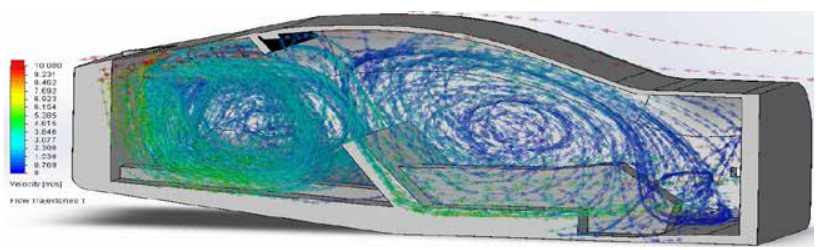

Figura 14. Flujo en la cabina con la Rejillas Louvers. Fuente: elaboración de los autores. [Solidworks ${ }^{\circledast}$ edición educativa (2012-2013)].

\section{Película térmica}

Una ganancia de calor por radiación solar a través de la burbuja al interior de la cabina de la aeronave T-90 Calima, puede tener un índice de radiación solar para la ciudad de Cali correspondiente a $5,2 \mathrm{KW} / \mathrm{m}^{2}$ y tener un promedio anual entre 4,5 y $5 \mathrm{KW} / \mathrm{m} 2$ según lo registrado por el Instituto de Hidrología Meteorología y Estudio Ambientales (2006).

Para analizar el comportamiento térmico se utilizó la película "prestige 70"desarrollada por (3M Company, 2012), cuyo coeficiente de sombra es de 0,58; por lo cual, permite el paso de gran cantidad de luz visible. Se realizaron los cálculos para determinar el coeficiente de ganancia de calor solar (ver Ecuación 1). En la Figura 15 se muestra el rechazo de calor por radiación solar.

$S H G C($ Coeficiente de ganancia de calor solar $)=\frac{S C_{P M M A}}{1.15}$ $S H G C=0.504$

En total la película térmica rechaza un 68,047\% de radiación solar, por tanto el calor incidente corresponde a 1,661 KW/m² (ver Ecuación 2).

$$
\begin{aligned}
& Q_{\text {solar ganancia }}=(S H G C) *\left(\text { Area }_{\text {encristalado }}\right) *\left(\text { Calor }_{\text {solar incidente }}\right) \\
& Q_{\text {solar ganancia }}=0.504 * 1.47 \mathrm{~m}^{2} * 1.661 \frac{\mathrm{KW}}{\mathrm{m}^{2}}=1.237 \mathrm{KW}
\end{aligned}
$$

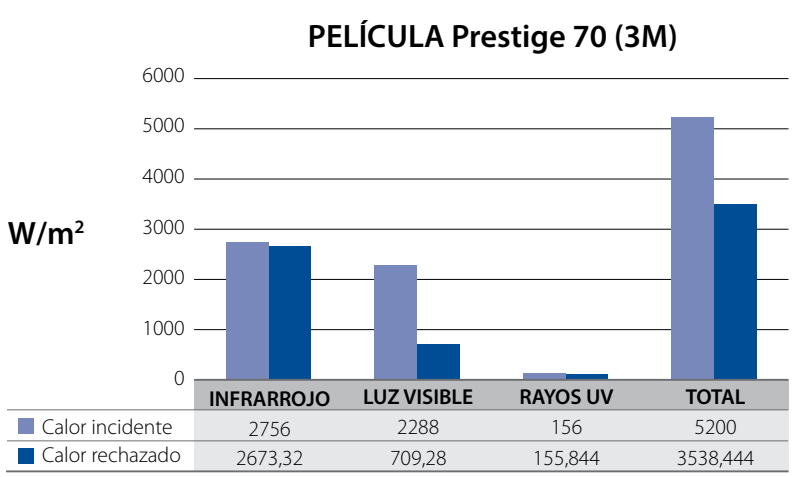

Figura 15. Rechazo de calor por radiación solar (3M Company, 2012). Fuente: elaboración de los autores.

Ante la evaluación de la película "Ceramic 70" (Eastman Chemical Company, 2014) el coeficiente de ganancia de calor dado y determinado por el fabricante, es de 0.52. En la Figura 16 se muestra el rechazo de calor por radiación solar.

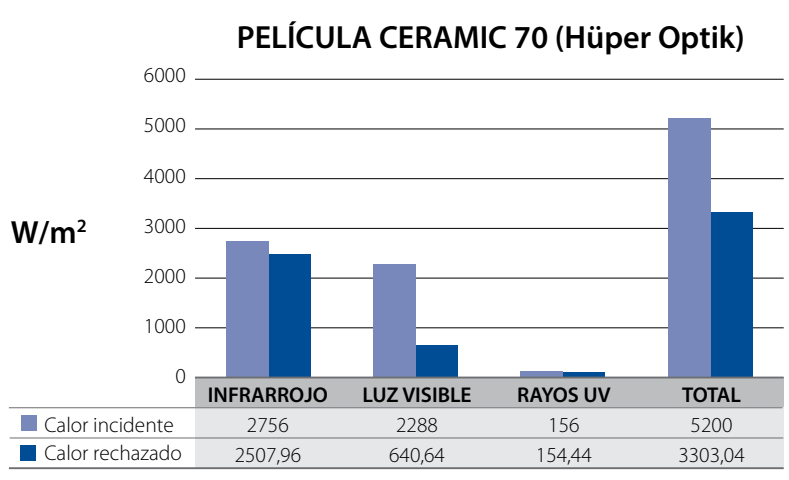

Figura 16. Rechazo de calor por radiación solar (Eastman Chemical Company, 2014)

Fuente: elaboración de los autores.

La película Ceramic 70 rechaza un 63,5\% de la radiación solar, por tanto la fracción de radiación que incide es de $1896,96 \mathrm{~W} / \mathrm{m}^{2}$ y la ganancia de calor solar 1,46 KW como se muestra en la Ecuación (3):

$$
\begin{aligned}
& Q_{\text {solar ganacia }}=0.52 * 1.478 \mathrm{~m}^{2} * 1.896 .96 \frac{\mathrm{KW}}{\mathrm{m}^{2}} \\
& Q_{\text {solar ganacia }}=1.46 \mathrm{KW}
\end{aligned}
$$

En comparación con la ganancia de calor solar sin incluir una película térmica, analíticamente se estima una reducción del 79.9\% para el caso descrito en la ciudad de Cali.

Debido a que las empresas generalmente no fabrican películas térmicas para superficies curvas como la burbuja de la aeronave, por tanto no es posible recubrirla en una sola pieza en el caso que si se pretende adherir una pelícu- 
la a la superficie interior de la carlinga, se debe hacer por secciones. La solución propuesta consistió en seccionar la película en la menor cantidad de partes posible.

Empleando el modelo a escala 1:28 proporcionado por la CIAC, se consiguió recubrir el área de la burbuja de la cabina empleando 6 secciones, se digitalizaron en varios croquis y posteriormente se reprodujeron a escala real, empleando el Plotter del laboratorio de diseño mecánico del Programa de Ingeniería Mecánica, de la Escuela Militar de Aviación "Marco Fidel Suárez" -EMAVI.

De la prueba realizada directamente sobre la burbuja de uno de los aviones de instrucción, se determinó que para recubrir la carlinga con una película térmica ésta se debe seccionar en 20 partes, con el propósito de minimizar efectos en el campo visual del piloto; en la Figura 17 se muestra los croquis de las secciones que recubrirán la carlinga de la aeronave.
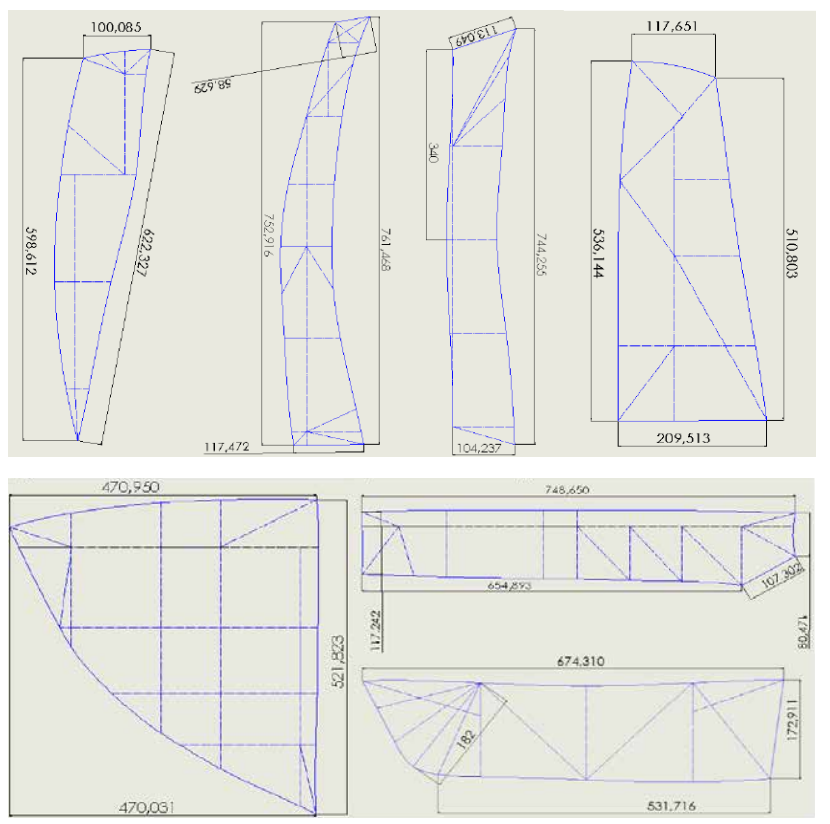

Figura 17. Croquis de las Secciones de la película térmica. Fuente: elaboración de los autores.

\section{Soporte de la carlinga para movimientos en tierra}

Debido a que el sistema de refrigeración por convección forzada implementado por la CIAC en el equipo FAC-2443 cumple parcialmente su función, se continúan realizando los movimientos en tierra con la carlinga desasegurada y entrecerrada, el problema radica en este método empleado, pues se usa el Canopy knife como se muestra en la Figura 18 para obstruir el cierre completo de la carlinga, generando de esta manera el riesgo como lesionar a uno de los tripulantes o caer hacia afuera de la cabina y por tanto imposibilitar la salida a vuelo de la aeronave, pues el Canopy knife se debe llevar a bordo de la aeronave en caso de presentarse alguna emergencia.

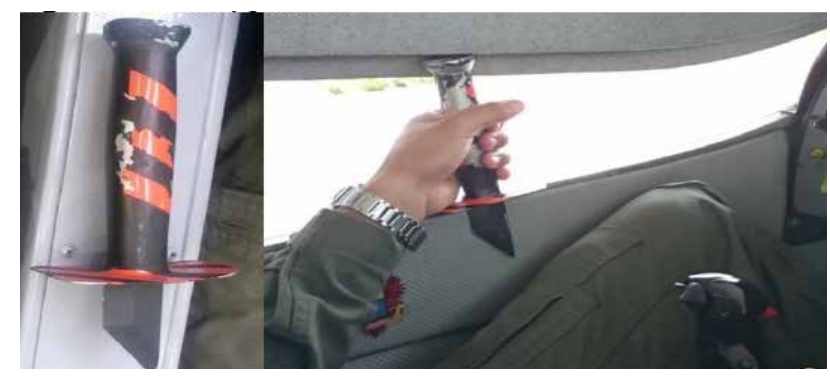

Figura 18. Canopy Knife.

Fuente: autores.

Por otra parte, el hecho de poner un elemento que obstruye el cierre de la carlinga solo de uno de los lados genera esfuerzos de torsión en las bisagras que sujetan el Canopy al fuselaje de la aeronave, influyendo negativamente en el alistamiento de las aeronaves, pues al presentarse las primeras fallas esto representa una necesidad de reajustar los programas de mantenimiento preventivo de la aeronave.

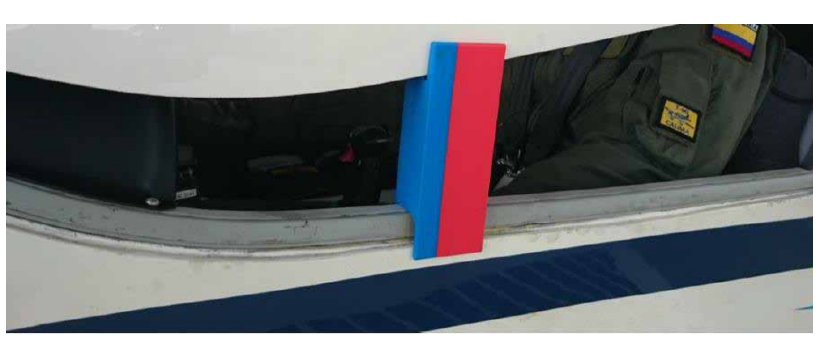

Figura 19. Ensayo del soporte.

Fuente: autores.

Por tal motivo, y con el objetivo de estandarizar un modo más adecuado y seguro para tal fin, se diseñó un soporte adaptado a la geometría diseñado en Solidworks ${ }^{\circledR}$ edición educativa (2012-2013) y se imprimió en la máquina de prototipado 3D del laboratorio de análisis estructural del programa de ingeniería mecánica en la EMAVI. El soporte preliminar se probó en varias aeronaves y queda sujeto entre el fuselaje y el borde de la carlinga como se muestra en la Figura 19.

\section{Conclusiones}

Con la implementación de estas propuestas se busca que la Fuerza Aérea Colombiana, siga siendo posicionada como una de las pioneras y promotoras del desarrollo de la Industria Aeronáutica de la región de la mano con la 
Corporación de la Industria Aeronáutica Colombiana, al lograr afrontar los problemas presentados en su producción del avión, inclusive los inconvenientes de temperatura en cabina, obteniendo un producto certificado, dando lugar a futuras negociaciones con otras naciones y/o entidades que manifiesten especial interés en adquirir la aeronave.

Para completar el ciclo de recirculación de aire se deben agregar salidas en la parte superior del compartimiento de equipaje donde se estanca el flujo de aire, y asimismo reducir el tiempo de renovación del aire, incrementando la velocidad del flujo hasta un promedio de $5 \mathrm{~m} / \mathrm{s}$ contribuyendo a mejorar la sensación térmica de los pilotos.

La implementación de películas térmicas reduce entre un $75 \%$ y $80 \%$ del calor transferido por la radiación solar al interior de la cabina, siendo un cambio notorio especialmente en movimientos en tierra cuando la radiación solar incidente y reflejada por la superficie de asfalto es máxima.

\section{Agradecimientos}

Los autores agradecen al Ing. Jorge Luis Pincay por sus aportes al trabajo de grado, al personal del Programa de Ingeniería Mecánica, al director de la CIAC señor General del Aire (RA) Flavio Enrique Ulloa Echeverry, al señor CT. Julián Andrés Delgado, a la señorita CT. Gina Galeano. Al Grupo de Entrenamiento de Vuelo, al señor MY. Julio Cesar Gonzáles, al señor CT. Jairo Andrés Perdomo, al TE. Cristian Andrés Russi. Al personal del Grupo Técnico, al TC. Héctor Fabio Garzón, al personal de Oficiales y Suboficiales del Grupo Académico de la Escuela Militar de Aviación "Marco Fidel Suárez".

\section{Referencias}

3M Company. (2012). Prestige Series 70. Retrieved from http:// solutions.3m.com/wps/portal/3M/en_US/Window_Film/ Solutions/Markets-Products/Residential/Sun_Control_Window_Films/Prestige_Series/

Acosta Palacios, F. E., y Cedeño Niño, N. F. (2015). Diseño del sistema de climatización de la cabina del avión de instrucción T-90C CALIMA. Escuela Militar de Aviación "Marco Fidel Suárez."

Alvin H. Sacks, J. R. S. (1951). Theoretical investigation of submerged inlets at low speeds. Washington, DC, United States. Retrieved from http://naca.central.cranfield.ac.uk/reports/1951/nacatn-2323.pdf

CIAC. (2015). Historia, Primara Aeronave. Retrieved February 19, 2015, from http://www.ciac.gov.co/productos-y-servicios/ historia/141

Eastman Chemical Company. (2014). Huüper Optik Nano-Ceramic
70 Window Film. Retrieved from https://www.huperoptik. com/en/news/detail.aspx?t=1\&id=27

Escuela Colombiana de Ingeniería. (2008). Curso de Higiene y Seguridad Industrial. Retrieved from http://www.escuelaing.edu. co/uploads/laboratorios/6299_temperatura.pdf

Establecimiento de Sanidad Militar (ESM). (2013). Informe de Mediciones Ambientales T-90. Santiago de Cali.

Evektor-Aerotechnik, A. (2015). Cosmik EV-97. Retrieved July 13, 2015, from http://www.evektor.cz/

Frick, C. W., Davis, W. F., Randall, L., \& Mossman, E. A. (1945). An Experimental Investigation of NACA Submerged-Duct Entrances. Washington, DC, United States. Retrieved from http://ntrs. nasa.gov/search.jsp?R=20090012113

Garmin Ltd. (2015). Garmin. Retrieved July 15, 2015, from http:// www.garmin.com/en-GB

Instituto de Hidrología Meteorología y Estudio Ambientales. (2006). Atlas de radiación solar de Colombia. Retrieved from http://www.si3ea.gov.co/Home/EnergiaSolar/tabid/74/language/en-US/Default.aspx

Mancera Fernández, M. J. (2016). Temperatura. Retrieved January 22, 2015, from http://www.manceras.com.co/publicaciones. htm

Naval Aerospace Medical Institute. (1991). Flight Surgeon `s Manual tercera edición. (U.S. Government Office., Ed.) (Tercera ed.). Washington D.C:

SolidWorks Corp. (2015). Solidworks edición educativa. Retrieved from http://www.solidworks.com/

Stephen Blee. (2011). Cosmik EV-97 TeamEuroStar UK. Retrieved July 13, 2015, from http://tinyurl.com/nk6svab

Susan C. Kim, MD - Pediatría \& John Pope, M. P. (2014). Temperatura corporal. Retrieved July 9, 2015, from http://www. uwhealth.org/spanishhealth/topic/medicaltest/temperatura-corporal/hw198785.html

U.S. Air Force Photo by Christian Turner. (2011). Flugzeugbau DG1001 Glider. Retrieved July 15, 2015, from http://www.edwards.af.mil/photos/mediagallery.asp?gallerylD=2515\&?id=1 \&page $=4 \&$ count $=48$ 


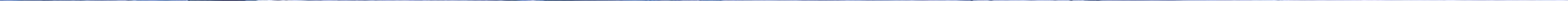

